\title{
On becoming a mother after cancer survival: a comprehensive literature review
}

\author{
Helena Sousa', Sónia Castro², M. Graça Pereira ${ }^{3}$ \\ 1 Center for Health Technology and Services Research (CINTESIS.UA), Department of Education and \\ Psychology, University of Aveiro, Campus Universitário de Santiago, 3810-193 Aveiro, Portugal \\ ${ }_{2}$ Breast Cancer Clinic/Psycho-Oncology Service, Francisco Gentil Portuguese Institute of Oncology of \\ Porto, Porto, Portugal \\ ${ }^{3}$ Health \& Family Research Group, School of Psychology, University of Minho, Braga, Portugal \\ Research Center in Psychology (CIPsi), School of Psychology, University of Minho, Braga, Portugal
}

Correspondence:

M. Graça Pereira School of Psychology, University of Minho, Campus de Gualtar, 4710 Braga, Portugal e-mail: gracep@psi.uminho.pt

Received: 24.05.2021 Accepted: 22.06.2021

DOI: 10.24292/01.OR.122220621 Copyright $\odot$ Medical Education. All rights reserved.

\section{ABSTRACT}

Problem identification: The desire for motherhood is one of the top priorities for most female young cancer survivors. The risk of infertility after cancer therapy is an important concern with several physical and emotional consequences. This comprehensive literature review aims to summarize and contextualize recent research that has been carried out on female fertility after cancer, suggesting future research and clinical directions.

Literature search: Searches included the key words "cancer", "female", "adults", and "fertility". Studies were selected if focused on infertility issues in female cancer survivors.

Data synthesis: Eight themes were identified: the effects of anticancer treatments on fertility, fertility preservation methods, international recommendations regarding infertility risk, health professionals' attitudes towards fertility preservation, patients' concerns regarding the risk of infertility, patients' information needs, and the impact of the risk of infertility in patients' quality of life.

Conclusions: Psycho-oncologists should be integrated in the oncofertility teams to assess patients' needs before cancer treatment initiation.

Key words: cancer, oncology, fertility, motherhood, survivorship, review 


\section{INTRODUCTION}

In developing countries, over the last decades, cancer is increasingly common in women of childbearing age [1, 2]. Breast cancer cervical cancer, thyroid cancer, non-Hodgkin's lymphoma, and leukemia are the most frequent cancers among women younger than 40 [3].

Due to advances in early diagnosis, improved treatment protocols, and individualized therapies, cancer survival rates are currently exceeding $80 \%$ for some of these cancer types [4]. These higher survival rates increase the importance placed on survivorship issues, such as infertility risk [5].

The desire for motherhood is one of the top priorities for most young female cancer survivors [6]. According to recent studies, $75 \%$ of women between 18 and 45 years old with a diagnosis of cancer wish to have children [7]. With the recent voluntary deferral of procreation, especially seen in Western societies, many women, at the time of diagnosis, have yet to initiate family or have not yet completed their family wishes. Although surrogacy and adoption can be an option, many women voice a preference for biological parenting [8].

Since thousands of cancer patients and survivors are in their reproductive age and the risk of infertility after cancer is a reality that these women must face, discussing fertility after cancer treatment is a subject of great clinical importance. Women should have the opportunity to plan the desired motherhood, given that several methods are already well-established and available [2].

In this context, oncofertility emerges as a clinical specialty that aims to understand cancer patients and survivors' needs regarding their reproductive potential before, during, and after antineoplastic treatments [9]. Given the international recommendations $[10,11]$, physicians should advocate for the timely provision of fertility information, for the patients' referral to fertility preservation methods [12], and for the importance of establishing oncofertility consults as a part of current medical practice in oncology centers $[10,11]$.

In the present paper, a comprehensive literature review is presented that intends to summarize and contextualize recent research that has been carried out on female fertility after cancer therapy, suggesting future research and clinical directions that aim to optimize patient psychosocial care during survival.

\section{METHODOLOGY}

Two authors (Sousa and Pereira) performed the search and carefully reviewed the published literature listed in PubMed and PsycINFO from January 2010 to December 2020. Hence, all searches included the key words "cancer", "female", "adults", and "fertility". Limits were applied for searches based on tittles and abstracts. The three authors (Sousa, Castro and Pereira) independently selected the most relevant articles considering this review's goals. Studies were selected if focused on the exploration of infertility issues in female cancer survivors. Other reviews on the topic were also considered and retrieved from the same databases. Articles were excluded if not written in English or Portuguese. All articles and its pertinence to the present review were discussed and a consensus was reached based on the most frequent themes that were found.

\section{RESULTS}

To make the search results easier to describe, this comprehensive review presents different subheadings: effects of anticancer treatments on fertility, fertility preservation methods, international recommendations regarding infertility risk, health professionals' attitudes towards fertility preservation, patients' concerns and attitudes regarding the risk of infertility, patients' information needs regarding fertility preservation, and the impact of the risk of infertility on patient's quality of life.

\section{EFFECTS OF ANTICANCER TREATMENTS ON FERTILITY}

The negative effect of cancer therapy on female fertility is well-known. Some chemotherapeutic agents, abdominal or pelvic radiation, bone marrow transplantation, and surgery for gynecological malignancies, have a high risk of gonadal damage [13]. Especially chemotherapy and endocrine treatments have experienced a significant increase in the last decade $[14,15]$. These antineoplastic treatments may partially or definitively affect ovarian function and lead to early menopause with all of its inherent risks, including infertility, that may be temporary or permanent [16].

Adjuvant chemotherapy, particularly with alkylating agents (e.g., cyclophosphamide, ifosfamide, chlorambucil, melphalan, busulfan, and procarbazine), poses the greatest risk of significant or permanent damages to female fertility [17]. These agents are responsible for high gonadotoxicity and amenorrhea in $18 \%$ to $61 \%$ of women under 40 [16]. In hormone-dependent breast cancer tumors, pre-menopausal women are also prescribed hormonal replacement therapy, such as tamoxifen [14]. In these cases, 
women are advised to postpone their pregnancy for a minimum of 2 to 5 years, since the risk of recurrence is higher in this period [18]. This hormonal treatment is proposed for a minimum of five years; nevertheless, extending tamoxifen up to ten years rather than stopping at five, has been proven to further reduce recurrence and mortality [19]. This extension has been incorporated into the international guidelines for cancer treatment preventing women from considering pregnancy, as fertility is also likely to be reduced due to age-related biological decline [20].

Nonetheless, these patients should not be discouraged to become pregnant, since recent studies suggested that pregnancy did not adversely affect survival in female cancer survivors [21]. Research also revealed that local or systemic anticancer treatments posed no further risks for the offspring of cancer survivors. However, there is a higher risk for miscarriage in these situations [22]; therefore, there are some necessary prerequisites that should be considered when supporting a pregnancy after cancer. The interval between treatment and conception is one of the most important considerations, since women who conceived less than one year after chemotherapy, had higher risks of preterm birth than women who conceived 2 years or more after treatment [23].

\section{FERTILITY PRESERVATION METHODS}

Increasing survival rates in female cancer survivors of reproductive age have led to the development and increased use of several fertility preservation techniques [2]. However, fertility preservation procedures are usually expensive and time-consuming [24]. Different techniques can be presented before treatment initiation, based on the patient's age and health status, as well as the risk of ovarian involvement [24].

Embryo cryopreservation is the most well-established option for female fertility preservation, as recognized by ASCO (American Society of Clinical Oncology) and ASRM (American Society for Reproductive Medicine) [10,11]. This procedure involves an ovary stimulation period with daily injections of gonadotrophins and ultrasonographic monitoring of follicle growth, which requires exposure to high levels of estrogen and a delay in systemic treatment commencement for at least 2 to 3 weeks. This method also implies that there is a source of male gametes, sometimes causing the exclusion of women who do not have a steady partner [24] In these situations, other viable options can be presented such as the cryopreservation of oocytes, which also requires an ovarian stimulation procedure, a subsequent follicular puncture, and cryopreservation obtained by vitrification. This last technique has significantly improved the survival of oocytes, fertilization rates, and the ratio of high-quality embryos due to slow freezing, allowing better conservation results [11].

In addition to these two methods, there are other experimental options, such as ovarian tissue cryopreservation, especially useful when hormonal stimulation is undesirable and/or there is urgency in anticancer treatment initiation. This technique involves a laparoscopic surgery for harvesting a fragment of the ovary. Hereafter, the ovarian tissue is properly prepared and cortex fragments are isolated for cryopreservation. Whenever necessary, the fragments are thawed and grafted on the remaining ovary orthotopic transplantation - or in another location - heterotopic transplantation. After transplantation, the ovarian tissue can restore its endocrine function and fertility [24]. Concerning gonadotropin-releasing hormone agonist ( $\mathrm{GnRHa})$, the evidence is still inconclusive regarding its isolated success for fertility preservation during chemotherapy [11].

ASCO has recently updated its guidelines for fertility preservation in cancer patients. When is clinically safe and the patient has time before treatment commencement, ASCO recommends that oocyte cryopreservation should be offered [11]. Besides, ovarian cryopreservation is another procedure with great potential in the near future [24].

Therefore, with the recent advances in biomedicine and technology, the probability of childbearing after aggressive antineoplastic treatments is constantly increasing. Nevertheless, fertility outcomes in oncology patients have not been adequately studied given the small percentage of patients that attempted to get pregnant after cancer. Many studies have reported good results of these procedures, but only a few of them reported pregnancy outcomes in patients returning to use their embryos or oocytes after cancer $[5,13]$.

\section{INTERNATIONAL RECOMMENDATIONS REGARDING INFERTILITY RISK}

ASCO recommendations regarding fertility preservation encourage oncologists to address the risk of infertility with their patients treated during childbearing years, to discuss fertility preservation options, and to refer them to reproductive specialists [11, 25].

First, patients should be informed about the feasibility of pursuing fertility preservation options and its dependency on each patient's recurrence risk, prognosis, and risk of infertility or early menopause from oncology therapy. Next, fertility preservation options should be discussed with their respective success rates, 
including those considered experimental. Clinicians should also explain that fertility procedures may be expensive and time-consuming and, therefore, imply antineoplastic treatment delay [11]. Thus, a prompt referral to a fertility specialist would optimize the time between diagnosis and cancer treatment commencement [25]. Meeting with a social worker may also be beneficial to support the decision-making process, to consider the financial resources and the associated costs of this procedure [26].

Although these guidelines represent a step forward, additional efforts are needed to encourage clinicians to include infertility risk as part of the standard discussion for all cancer patients within reproductive years. This topic is not consistently addressed in clinical practice, despite the aforementioned ASCO recommendations $[11,26]$, and the provision of fertility preservation techniques is still lacking. Improvements need to be made in the number of referrals from oncology to reproductive medicine specialists [25].

\section{HEALTH PROFESSIONALS' ATTITUDES TOWARDS FERTILITY PRESERVATION}

Many factors impact patients' access to fertility preservation information. A few studies explored health professionals' concerns regarding fertility preservation and, although evidence suggested greater awareness, knowledge, and willingness to discuss this topic with patients, many patients still report receiving little information $[27,28]$. In this sense, a recent study reported that only $49.5 \%$ of physicians routinely informed their patients of childbearing age about the risk of infertility after cancer treatment [29].

Several barriers were identified regarding communication concerning fertillity preservation, namely the clinician insufficient knowledge regarding the available fertility preservation options, the lack of communication and access to reproductive specialists, and patients' characteristics (e.g., prognosis, age, parental status, marital status, financial capacity, sexual orientation, sexual maturity, and whether or not the patients initiate the conversation) [28]. Also, the clinicians' perception of fertility as a minor issue, the fear of disease aggravation due to anticancer treatment delay, the availability of educational materials, as well as legal and ethical issues such as the subsequent use of male gametes, were some of the additional barriers reported [28]. However, the amount of time spent with patients was described as one of the strongest barriers to discuss fertility issues with female cancer survivors [30].

These informational and communication barriers may prejudice and bias the patient's decision-making processes [31]. Thus, it is of utmost importance to overcome the intrinsic barriers justifying why oncologists do not discuss fertility risk issues with their patients, namely the lack of communication skills [30], and the oncologists' perceptions of patients' characteristics as dictating their willingness to undergo fertility preservation.

Future work should ensure that health care professionals receive adequate training on how to discuss fertility risk and preservation options with young patients and their partners. Comprehensive counseling should also include related issues such as contraception use and the health implications of early menopause [32].

Nevertheless, it is also important to recognize that the discussion of fertility prognosis and risk of recurrence at the time of diagnosis, may become an additional burden for clinicians. Communication skills should also be trained by consulting with other health providers, such as clinical psychologists.

\section{PATIENTS' CONCERNS AND ATTITUDES REGARDING} THE RISK OF INFERTILITY

Fertility-related psychological distress persists from diagnosis through survivorship. Previous research suggested that female cancer survivors presented high rates of uncertainty, worry, stress, anger, anxiety, nervousness, and fear related to the unknown fertility status [33, 34]. Moreover, some patients report constant and persistent reproductive concerns that disrupt their life purpose with the knowledge that cancer may affect their lives forever [35].

Several studies focused on exploring the sociodemographic and clinical characteristics related to the risk of infertility, revealing that younger patients, highly educated, unmarried, professionally active, childless, who wish to have children and who previously had trouble getting pregnant before cancer, are the most concerned about the impact of anticancer treatments on fertility [36]. Young and childless women revealed a higher desire to become pregnant after breast cancer compared to women who already had children and who seemed to be more concerned about the possibility of recurrence [37, 38]. Regarding clinical variables, studies were mainly focused on time since diagnosis, cancer stage, disease dissemination and the quality of the information received, as factors correlated with infertility concerns and willingness to undergo fertility preservation procedures. Thus, when dealing with a cancer diagnosis and, especially in a situation of early-stage cancer, women revealed to be less concerned about fertility preservation, while being more focused on anticancer treatment and survival $[37,39]$. Considering the disease stage, 
studies hypothesized that higher cancer stages (and, therefore, with a higher risk of recurrence) were associated with greater acceptance of cancer treatments and its side effects [37]. Even so, fertility is important regardless of cancer stage and, therefore, the opportunity for preservation should be openly discussed with these patients as well [11].

Regarding patients' attitudes towards fertility preservation, the European Organization for Research and Treatment of Cancer developed a study aiming to evaluate the proportion of participants who would not agree to chemotherapy if it affected their fertility. The authors found that, although survival and cure were the priority for young women with cancer, fertility preservation emerged as a matter of great importance. Results revealed that $59 \%$ of women wished to have children (or more children), compared to $36 \%$ of women who do not want to have any more children for fear of recurrence. Women who had an easier acceptance of the risk of infertility were women who already had children, did not intend to have more children, who were still waiting for the beginning of treatment (the initial stage of the disease), and living in Western Europe [37].

In general, female patients revealed more positive than negative attitudes towards fertility preservation, which leads to the hypothesis that they are receptive to decide in favor of fertility preservation procedures when correctly informed.

\section{PATIENT INFORMATION NEEDS REGARDING FERTILITY PRESERVATION}

Research consistently reports that patients are generally uninformed about fertility preservation procedures, presenting low levels of literacy regarding this medical issue [27]. In a recent study, $43 \%$ to $62 \%$ of female cancer survivors reported important unmet information needs, $26 \%$ felt that infertility risk was not well addressed by their doctors and revealed to be dissatisfied with the received information [32]. Also, feeling overwhelmed with their cancer diagnosis, being unaware of the possible treatment impact on fertility, and the costs associated with these procedures, were three important reasons for not undergoing fertility preservation [27]. Moreover, several female patients revealed never discussing this topic with their husband, family, friends, and/or health professionals because they felt that infertility risk was devalued and considered a minor issue when facing cancer [34, 38-40]. Additionally, patients older than 35 years and with children were less likely to be informed about preservation options, and several women did not recall having these conversations with their clinicians $[27,40]$.
There is a consistent need to inform patients about the impact of the anticancer treatment on fertility and the existing preservation methods, regardless of their sociodemographic and clinical characteristics. Furthermore, some patients felt that there was a bias between the information that was delivered and the relevance the clinicians gave to the subject. Other patients revealed feeling pressured to start anticancer treatment and, therefore, not having enough time to make an informed decision [41]. This pressure could be the reflection of a judgment-bias regarding the clinicians' perceptions when considering the importance patients gave to their fertility, after surviving cancer, which emphasizes the importance of how the information was delivered that could have impacted the patient's decision-making processes. Moreover, the need for more information seems to be less prominent at diagnosis, but increases during and after systemic anticancer treatments [42]. After treatment, patients seem to recognize the importance of discussing fertility before the beginning of the treatment and revealed some disappointment regarding the quality and quantity of the information received [42]. Although fertility was not the priority at diagnosis, some women revealed regret for not choosing to consider preservation when it was possible. Research on decisional regret confirmed that women's perspectives on fertility change over time, from the perception that procreation was not important to feeling regret over not having pursued conservation [42]. These studies also revealed that pre-treatment fertility satisfactory counseling leads to lower levels of post-treatment regret and better quality of life [43].

Lack of information regarding fertility risks can have important psychosocial consequences [42], and lead to patient's uncertainty, depression, anxiety, distress, anger, and confusion [1, 12]. In this sense, fertility counseling has a fundamental role in assisting with fertility decision-making, but also in supporting patients with the psychological distress caused by the uncertainty of infertility [12]. More research is needed to understand what are the decision-making factors underlying fertility preservation. Research available is mostly cross-sectional and retrospective and there is a lack of longitudinal studies that may contribute to better knowledge about patients' concerns, needs, and attitudes regarding infertility risks, as well as how it evolves. Research should also focus on exploring individual risk factors for decision-regret in women with cancer of childbearing age and what are their future expectations regarding their reproductive capability and family plans. Only then, clinicians will have clear guidelines on how to educate patients about fertility preservation alerting for the possibility of mind-changing processes, while encouraging the discussion of situations that might not seem urgent at the moment of diagnosis but will affect the long-term quality of life. 


\section{IMPACT OF THE RISK OF INFERTILITY ON THE} PATIENT'S QUALITY OF LIFE

Recent studies indicated that the potential for fertility loss may be more stressful than a cancer diagnosis itself [1]. Infertility seems to be associated with high levels of distress, anxiety, and depression [12], and lower quality of life [1]. It has also been identified as an experience marked by feelings of grief and loss, even in women who have fulfilled their family wishes before cancer [22]. Especially young and childless women, revealed higher levels of psychological distress, lower self-esteem, uncertainty, relationship problems, more intrusive thoughts, and more avoidance strategies $[1,12,25,42]$, regardless of the cancer site.

It is important to mention that some studies revealed that even women who already had children and who did not think about having more children after cancer, also face the risk of fertility loss as an emotionally draining experience $[22,25,42]$. Even though $70 \%$ of patients revealed that the disease had no impact on their desire to have children, $13 \%$ to $15 \%$ stated that cancer diagnosis increased their desire to be mothers again and the value given to parenting [42].

In summary, few studies have evaluated the impact of fertility on female survivors' quality of life, including childhood cancer survivors. Little is known about the psychological impact of infertility risk during and after cancer treatment, or which individual variables can potentially protect or impact the quality of life in these situations. Infertility in young women with cancer is another setback to their personal and relational development considered another great loss caused by cancer. Therefore, it is important to better understand the impact of infertility in patients' overall functioning, so that strategies can be developed to facilitate doctor/patient's communication processes and patients informed decision-making.

\section{LIMITATIONS}

A comprehensive literature review has some limitations that need to be acknowledged. This type of study is not as exhaustive as a systematic review. In this sense, a strict protocol was not followed and studies were selected based on their relevance regarding the theme of this review. No inclusion or exclusion criteria regarding population, design, or outcomes were explicitly considered for the selection of studies. Instead, the authors adopted a broader perspective and analyzed the studies retrieved from the databases based on their pertinence and team consensus. This increases the possibility of selection bias. In addition, only female fertility was considered. Future studies should focus on summa- rizing recent data regarding male fertility issues, since an update on this matter is also needed.

\section{CONCLUSION}

Several studies confirmed the importance of biological parenting for female cancer patients of reproductive age. This comprehensive review emphasized that the risk of infertility exceeds the diagnostic and clinical dimension of cancer, and is of great significance for female patients since it endangers a fundamental life goal for most women: the opportunity of biological motherhood which has several psychological, social, and spiritual implications for the couple and the family.

The risk of infertility seems to increase the psychological distress associated with a cancer diagnosis, regardless of the patients' parental status, since it interferes with women's ability to decide about their life project regarding reproducibility and was imposed by the disease process, instead of decision-making. Therefore, even patients who did not intend to have children before the diagnosis can feel the loss and the anger associated with infertility risk as a result of cancer and its treatment. Since fertility is usually described as an important part of the definition of being a woman, all patients should be informed about infertility risk due to anticancer treatment, regardless of whether or not they have decided to have more children in the future.

Future studies should also identify an optimal approach to include fertility counseling and support resources into patients and survivor's care programs. Infertility risk should also be included in informed consent about anticancer treatment, especially before chemotherapy. Therefore, there is a need to develop informational materials, clinical guidance, and multidisciplinary consultations to facilitate communication between physicians and patients. More information is also needed on which individual and contextual factors might influence female cancer survivors' decision-making to undergo or not fertility preservation. Studies are also needed to assess and characterize young cancer patients' concerns about their infertility risk, their expectations of fertility, their informational needs, and attitudes towards childbearing during and after anticancer treatment.

\section{IMPLICATIONS FOR PSYCHOSOCIAL ONCOLOGY}

Health professionals should not make assumptions regarding fertility, since studies consistently indicate that patients, regardless of their clinical and sociodemographic characteristics, assess positively the possibility of discussing this issue with their oncologist. 
As a result, patients become more satisfied with the health care they received, regardless of choosing or not to preserve fertility.

Additionally, an oncologist should consider that discussing infertility risk with patients does not decrease treatment adherence and, therefore, all the risks, options and benefits, should be clearly and carefully addressed, ensuring that the patient makes an informed decision. Although patients may be more focused on their diagnosis, oncologists should advise patients regarding potential fertility threats, present them with different options, and prevent future regrets.
Fertility should be addressed as a fundamental dimension of female cancer treatment, in a multidisciplinary approach designed to be implemented as a routine practice. In this sense, psycho-oncologists should be integrated in the oncofertility teams to assess patients' needs before treatment initiation. Several issues should be identified and addressed, namely the impact of the infertility risk on couple and family dynamics, communication, the bereavement process, the changes in the individual and family identity and, finally, the timely management of other alternatives to biological motherhood.

\section{References}

1. Assi J, Santos J, Bonetti T et al. Psychosocial benefits of fertility preservation for young cancer patients. J Assist Reprod Genet. 2018; 35(4): 601-6. http:// doi.org/10.1007/s10815-018-1131-7.

2. Goeckenjan M, Freis A, Glaß K et al. Motherhood after cancer: fertility and utilization of fertility-preservation methods. Arch Gynecol Obstet. 2020; 301(6): 1579-88. http://doi.org/10.1007/s00404-020-05563-w.

3. Fidler MM, Gupta S, Soerjomataram I et al. Cancer incidence and mortality among young adults aged 20-39 years worldwide in 2012: a population-based study. Lancet Oncol. 2017; 18(12): 1579-89. http://doi.org/10.1016/s1470-2045(17)30677-0.

4. Allemani C, Matsuda T, Di Carlo V et al. Global surveillance of trends in cancer survival 2000-14 (CONCORD-3): analysis of individual records for 37513025 patients diagnosed with one of 18 cancers from 322 population-based registries in 71 countries. Lancet. 2018; 391(10125): 1023-75. http:// doi.org/10.1016/50140-6736(17)33326-3.

5. Anderson RA, Brewster DH, Wood R et al. The impact of cancer on subsequent chance of pregnancy: a population-based analysis. Hum Reprod. 2018; 33(7): 1281-90. http://doi.org/10.1093/humrep/dey216.

6. Abdel-Razeq HN, Mansour RA, Ammar KS et al. Amenorrhea, fertility preservation, and counseling among young women treated with anthracyclines and taxanes for early-stage breast cancer, a retrospective study. Medicine. 2020; 99(11): e19566. http://doi.org/10.1097/MD.0000000000019566.

7. La Rosa VL, Shah M, Kahramanoglu I et al. Quality of life and fertility preservation counseling for women with gynecological cancer: an integrated psychological and clinical perspective. J Psychosom Obstet Gynaecol. 2020;41(2): 86-92. http://doi.org/10.1080/0167482X.2019.1648424.

8. Gorman JR, Julian AK, Roberts SA et al. Developing a post-treatment survivorship care plan to help breast cancer survivors understand their fertility. Support Care Cancer. 2018; 26(2): 589-95. http://doi.org/10.1007/s00520-017-3871-9.

9. Woodruff TK. The Oncofertility Consortium - addressing fertility in young people with cancer. Nat Rev Clin Oncol. 2010; 7(8): 466-75. http://doi. org/10.1038/nrclinonc.2010.81.

10. Loren AW, Mangu PB, Beck LN et al. Fertility preservation for patients with cancer: American Society of Clinical Oncology clinical practice guideline update. J Clin Oncol. 2013; 31(19): 2500-10. http://doi.org/10.1200/JCO.2013.49.2678.

11. Oktay K, Harvey BE, Partridge AH et al. Fertility Preservation in Patients With Cancer: ASCO Clinical Practice Guideline Update. J Clin Oncol. 2018; 36(19): 1994-2001. http://doi.org/10.1200/JCO.2018.78.1914.

12. Logan S, Perz J, Ussher J et al. Clinician provision of oncofertility support in cancer patients of a reproductive age: A systematic review. Psychooncology. 2018; 27(3): 748-56. http://doi.org/10.1002/pon.4518.

13. Alvarez RM, Ramanathan P. Fertility preservation in female oncology patients: the influence of the type of cancer on ovarian stimulation response. Hum Reprod. 2018; 33(11): 2051-9. http://doi.org/10.1093/humrep/dew158.

14. Burgos N, Cintron D, Latortue-Albino $P$ et al. Estrogen-based hormone therapy in women with primary ovarian insufficiency: a systematic review. Endocrine. 2017; 58(3): 413-25. http://doi.org/10.1007/s12020-017-1435-x.

15. Stumpf U, Kostev K, Siebenbürger G et al. Influence of chemotherapy and endocrine treatment on fractures in postmenopausal women with breast cancer - a retrospective cohort study. J Bone Oncol. 2020; 22: 100292. http://doi.org/10.1016/j.jbo.2020.100292.

16. Spears N, Lopes F, Stefansdottir A et al. Ovarian damage from chemotherapy and current approaches to its protection. Hum Reprod Update. 2019; 25(6): 673-93. http://doi.org/10.1093/humupd/dmz027.

17. Vassilakopoulou $M$, Boostandoost $E$, Papaxoinis $G$ et al. Anticancer treatment and fertility: Effect of therapeutic modalities on reproductive system and functions. Crit Rev Oncol Hematol. 2016; 97: 328-34. http://doi.org/10.1016/j.critrevonc.2015.08.002.

18. Ter Welle-Butalid MEE, Vriens IJHI, Derhaag JGJ et al. Counseling young women with early breast cancer on fertility preservation. J Assist Reprod Genet. 2019; 36(12): 2593-604. http://doi.org/10.1007/s10815-019-01615-6.

19. Davies $\mathrm{C}, \mathrm{Pan} \mathrm{H}$, Godwin $\mathrm{J}$ et al. Long-term effects of continuing adjuvant tamoxifen to 10 years versus stopping at 5 years after diagnosis of oestrogen receptor-positive breast cancer: ATLAS, a randomised trial. Lancet. 2013; 381(9869): 805-16. http://doi.org/10.1016/S0140-6736(12)61963-1.

20. Ben Charif A, Bouhnik AD, Rey D et al. Satisfaction with fertility- and sexuality-related information in young women with breast cancer - ELIPPSE40 cohort. BMC Cancer. 2015; 15: 572. http://doi.org/10.1186/s12885-015-1542-0.

21. Iqbal J, Amir E, Rochon PA et al. Association of the timing of pregnancy with survival in women with breast cancer. JAMA Oncol. 2017; 3(5): 659-65. http://doi.org/10.1001/jamaoncol.2017.0248.

22. Jardim FA, Lopes-Júnior LC, Nascimento LC et al. Fertility-related concerns and uncertainties in adolescent and young adult childhood cancer survivors. J Adolesc Young Adult Oncol. 2021; 10(1): 85-91. http://doi.org/10.1089/jayao.2020.0058. 
23. Hartnett KP, Mertens AC, Kramer MR et al. Pregnancy after cancer: does timing of conception affect infant health? Cancer. 2018 ; $124(22): 4401-7$. http://doi.org/10.1002/cncr.31732.

24. Rajabi Z, Aliakbari F, Yazdekhasti H. Female Fertility Preservation, Clinical and Experimental Options. J Reprod Infertil. 2018; 19(3): 125-32.

25. Lambertini M, Peccatori FA, Demeestere I et al. Fertility preservation and post-treatment pregnancies in post-pubertal cancer patients: ESMO Clinical Practice Guidelines ${ }^{\dagger}$. Ann Oncol. 2020; 31(12): 1664-78. http://doi.org/10.1016/j.annonc.2020.09.006.

26. Angarita AM, Johnson CA, Fader AN et al. Fertility Preservation: A Key Survivorship Issue for Young Women with Cancer. Front Oncol. 2016 ; 6: 102. http://doi.org/10.3389/fonc.2016.00102.

27. Mahey R, Kandpal S, Gupta M et al. Knowledge and awareness about fertility preservation among female patients with cancer: a cross-sectional study. Obstet Gynecol Sci. 2020; 63(4): 480-9. http://doi.org/10.5468/ogs.20003.

28. Vindrola-Padros C, Dyer KE, Cyrus J et al. Healthcare professionals' views on discussing fertility preservation with young cancer patients: a mixed method systematic review of the literature. Psychooncology. 2017; 26(1): 4-14. http://doi.org/10.1002/pon.4092.

29. Zhang H, Wang G, Jiang B et al. The Knowledge, Attitude, and Self-Reported Behaviors of Oncology Physicians Regarding Fertility Preservation in Adult Cancer Patients. J Cancer Educ. 2020; 35(6): 1119-27. http://doi.org/10.1007/s13187-019-01567-6.

30. Melo C, Fonseca A, Silva $C$ et al. Portuguese oncologists' practices regarding female fertility preservation: Which barriers most relate to these practices? Eur J Cancer Care. 2018; 27(2): e12812. http://doi.org/10.1111/ecc.12812.

31. Norton W, Wright E. Barriers and Facilitators to Fertility-Related Discussions with Teenagers and Young Adults with Cancer: Nurses' Experiences. J Adolesc Young Adult Oncol. 2020; 9(4): 481-9. http://doi.org/10.1089/jayao.2019.0092.

32. Benedict C, Nieh JL, Hahn AL et al. "Looking at future cancer survivors, give them a roadmap": addressing fertility and family-building topics in post-treatment cancer survivorship care. Support Care Cancer. 2021; 29(4): 2203-13. http://doi.org/10.1007/s00520-020-05731-3.

33. Logan S, Anazodo A. The psychological importance of fertility preservation counseling and support for cancer patients. Acta Obstet Gynecol Scand. 2019; 98(5): 583-97. http://doi.org/10.1111/aogs.13562.

34. Wettergren L, Ljungman L, Micaux Obol C et al. Sexual dysfunction and fertility-related distress in young adults with cancer over 5 years following diagnosis: study protocol of the Fex-Can Cohort study. BMC Cancer. 2020; 20(1): 722. http://doi.org/10.1186/s12885-020-07175-8.

35. Ussher JM, Perz J; Australian Cancer and Fertility Study Team (ACFST). Threat of biographical disruption: the gendered construction and experience of infertility following cancer for women and men. BMC Cancer. 2018; 18(1): 250. http://doi.org/10.1186/s12885-018-4172-5.

36. Sobota A, Ozakinci G. Determinants of fertility issues experienced by young women diagnosed with breast or gynaecological cancer - a quantitative, cross-cultural study. BMC Cancer. 2018; 18(1): 874. http://doi.org/10.1186/s12885-018-4766-y.

37. Senkus E, Gomez H, Dirix L et al. Attitudes of young patients with breast cancer toward fertility loss related to adjuvant systemic therapies. EORTC study 10002 BIG 3-98. Psychooncology. 2014; 23(2): 173-82. http://doi.org/10.1002/pon.3384.

38. Hawkey AJ, Ussher JM, Perz J et al. The impact of cancer-related fertility concerns on current and future couple relationships: People with cancer and partner perspectives. Eur J Cancer Care. 2021; 30(1): e13348. http://doi.org/10.1111/ecc.13348.

39. Ljungman L, Ahlgren J, Petersson LM et al. Sexual dysfunction and reproductive concerns in young women with breast cancer: Type, prevalence, and predictors of problems. Psychooncology. 2018; 27(12): 2770-7. http://doi.org/10.1002/pon.4886.

40. Garg R, Rebić N, De Vera MA. Information needs about cancer treatment, fertility, and pregnancy: qualitative descriptive study of reddit threads. JMIR Cancer. 2020; 6(2): e17771. http://doi.org/10.2196/17771.

41. Dagan E, Modiano-Gattegno S, Birenbaum-Carmeli D. "My choice": breast cancer patients recollect doctors fertility preservation recommendations. Support Care Cancer. 2017; 25(8): 2421-8. http://doi.org/10.1007/s00520-017-3648-1.

42. Armuand G, Wettergren L, Nilsson J et al. Threatened fertility: A longitudinal study exploring experiences of fertility and having children after cancer treatment. Eur J Cancer Care. 2018; 27(2): e12798. http://doi.org/10.1111/ecc.12798.

43. Benedict C, Thom B, Friedman DN et al. Fertility information needs and concerns post-treatment contribute to lowered quality of life among young adult female cancer survivors. Support Care Cancer. 2018; 26(7): 2209-15. http://doi.org/10.1007/s00520-017-4006-z.

Authors' contributions: Helena Sousa: study design, data analysis, data interpretation, search and data collection, manuscript preparation and manuscript review; M. Graça Pereira: study design, data analysis, data interpretation; manuscript preparation and manuscript review; Sónia Castro: manuscript preparation and manuscript review.

Conflict of interests:

Authors declare no conflict of interest.

Financial support: There was no financial support.

Ethics:

The authors had full access to the data and take full responsibility for its integrity. All authors have read and agreed with the content of the manuscript as written. The paper complies with the Helsinki Declaration, EU Directives and harmonized requirements for biomedical journals. 